\title{
49. THE INTERPLANETARY PLASMA AND THE HELIOSPHERE (PLASMA INTERPLANÉTAIRE ET DE L'HÉLIOSPHÈRE)
}

PRESIDENT: W. I. Axford.

VICE-PRESIDENT: A. Hewish.

ORGANIZING COMMITTEE: J. E. Blamont, A. Z. Dolginov, J. Geiss, A. Hundhausen, S. Grzedzielsky.

\section{INTRODUCTION}

This Commission was set up during the 1972 General Assembly to study the following problems: (1) the region where the solar wind joins the Sun (i.e. the solar corona-chromosphere interface); (2) the zodiacal light as it pertains to the solar wind; (3) radio observations of the solar wind and outer corona (especially interplanetary scintillations); (4) the heliosphere and interaction of the solar wind with the interstellar medium; (5) direct observations of the solar wind as far as they pertain to the above and to questions of an astrophysical nature (especially the composition of the sun, mass and angular momentum loss from stars, etc).

It is intended that this Commission should cater to the interests of astronomers and astrophysicists who are concerned with the above problems while recognizing that these and related topics are also of interest to members of other commissions and of other unions. We intend to stress the astrophysical aspects of the solar wind rather than the geophysical effects or solar-terrestrial relations.

\section{ORIGIN AND PHYSICAL PROCESSES IN THE SOLAR WIND (J. V. Hollweg)}

\section{A. Introduction}

It is perhaps remarkable that, in spite of continuous observational and theoretical effort since Parker's first discussion of the solar wind expansion (Parker, 1958), the solar wind remains a phenomenon which is understood only in the coarsest sense, and whose origin and internal physical processes still remain to be convincingly defined in detail. Even the most fundamental concepts, which are the sine qua non of any complete theoretical model of the solar wind, are at present either unknown, or known only with an accuracy which is insufficient to yield satisfactory agreement between theory and observations; among these fundamental concepts may be listed: (i) The solar sources of mass, momentum and energy to the corona and solar wind; (ii) the regions of the corona from which the solar wind originates; (iii) the coronal boundary conditions necessary for a realistic theoretical model of the solar wind expansion; (iv) the geometry of the solar wind flow; (v) the importance of MHD waves of solar origin in heating and accelerating the solar wind; (vi) the correct physical nature of the electron energy equation, including the physics of electron heat conduction and electron-ion thermal coupling; (vii) the physical processes responsible for the non-thermal distribution functions which are observed for electrons, protons and heavy ions in the solar wind.

The present difficulties in understanding the solar wind flow are of non-trivial consequence for a number of areas of broader astrophysical interest. The role of the solar wind in the evolution of our solar system can certainly not be properly evaluated until we are confident of our understanding of the solar wind as observed today. A similar comment applies also to the role of stellar winds in the evolution and present properties of other stars. Moreover, we must regard our understanding of the properties of a wide variety of astrophysical plasmas and flows as incomplete at least until we are confident of our understanding of the physics of the 
interplanetary and coronal plasmas. In addition, it may for some purposes be useful to develop a capability for predicting, from solar observations, the state of the solar wind at the earth (for example, in order to predict a variety of atmospheric effects which may be linked to the interaction between the solar wind and the magnetosphere), and such a predictive capability will require a more advanced theoretical understanding of solar wind physics than is available at present.

The following paragraphs present highlights of theoretical progress during the past several years, but also emphasized are the remaining problems, and possible progress which may be expected in the coming years. Section B considers the solar wind as it originates near the Sun, while Section $C$ discusses a number of physical processes occuring in the subsequent expansion into interplanetary space.

\section{B. Origin}

\section{The Corona}

The solar wind exists in virtue of the hot rarefied coronal plasma, which leads via the high thermal conductivity of the electron gas to an extended atmosphere which can not be statically contained by the interstellar plasma or magnetic field in the neighbourhood of the Sun. This statement is the essence of Parker's original discovery of the solar wind, and it has subsequently been greatly expanded upon (e.g., Hundhausen, 1972). But such a statement suffers from implicitly treating the corona merely as a boundary condition on the solar wind, and relegating its temperature and density to being determined by unknown physical processes which occur below some arbitrary lower boundary of the solar wind. Such an approach is not without practical advantages, but it would clearly be preferable to at least treat the solar wind, the lower corona and the corona-chromosphere transition region as a unit whose physical properties are determined by specified sources of mass, momentum and energy at the solar surface.

Such an approach can not be developed at present, however, because the sources of mass, momentum and energy are not yet conclusively identified. The mass source is perhaps the least serious problem, because the spicules can easily provide the solar wind mass flow (e.g., Beckers, 1972), but observational proof is lacking. The momentum source can only be specified in conjunction with the energy and mass sources, but it should be pointed out that some authors have presented coronal models which can not be self-consistent, because they include only a source of energy while ignoring the corresponding momentum. The energy source is generally regarded to be waves or shocks which originate from turbulent motions at the top of the convection zone (e.g., Stein and Leibacher, 1974), but observational proof of this important hypothesis is unfortunately lacking. There thus still remains a great deal of room for speculation and observation as to mass, momentum and energy transfer from the solar surface to the corona and solar wind. The speculative possibilities seem boundless, but it may be hoped that observations with increasing time and space resolution in the optical and UV regions may eventually show the putative waves and shocks which are heating the corona, while radio observations in the lower corona, as well as observations of heavy ions in the solar wind at $1 \mathrm{AU}$ (see Section C.I), may provide clues to an assortment of microscopic processes occurring in the coronal plasma.

Although the lack of a definitive identification of the sources of mass, momentum and energy hinder the development of a unified theory of the solar wind, lower corona and transition region, some interesting work has nevertheless been done. Pneuman (1973) and Noci (1973) have illuminated the effects of magnetic field geometry on the thermal and mass structure of the corona, while Endler (1975) and others have attempted to produce hot coronas by numerically solving the conservation equations with a specified model wave source at the solar surface. This latter approach tends to produce rather cool coronas, however, which may indicate that the electron heat conduction in the transition region is incorrectly understood; such a situation would not be surprising, since the electron heat conduction in the solar wind still remains to be properly described (Section C.II). 


\section{Coronal Holes}

One of the significant advances of the past several years is the realization that the solar wind flow, and particularly the high-speed streams, most probably originates in the coronal holes (Neupert and Pizzo, 1974). The arguments in favor of this point of view are: (i) correlations between the passage of coronal holes across the solar disc and passage of high-speed streams at the Earth; (ii) the coronal holes correspond to regions of open magnetic field which are most favorable for the solar wind expansion; (iii) the observed lower density and temperature of the coronal holes can be readily explained as being due to the loss of mass and energy to the solar wind.

Apart from the obvious advantages associated with knowing where the solar wind comes from, there is a less obvious theoretical advantage associated with the identification of coronal holes as the source of the solar wind. The lower density and temperature in a coronal hole probably place the solar wind in a regime in which it is easier to produce high temperatures and flow speeds at $1 \mathrm{AU}$ than would be the case if the solar wind originated from denser and hotter parts of the corona; the reason is simply that the lower mass density in a coronal hole is easier to heat and accelerate (Durney, 1973). This is a significant result, because a recurring problem in the solar wind has been to explain the observed high proton temperatures and flow speeds at $1 \mathrm{AU}$. But this is only a step in the right direction; a variety of other unsolved problems remains.

A significant disadvantage associated with the identification of coronal holes as the source of the solar wind is the resultant uncertainty in the boundary conditions which are needed to specify the solar wind flow. The very low density of a coronal hole makes it essentially invisible, and thus its density and temperature can only be guessed. At present it can only be stated that both density and temperature are lower in a coronal hole than elsewhere in the corona, but it should be pointed out that the lower temperature is only surmised from UV observations in the upper part of the corona-chromosphere transition region (Munroe and Whithbroe, 1972), and not above the region of coronal heating, which is the preferred location for applying the boundary conditions to the solar wind equations. This uncertainty in the boundary conditions has the important consequence that conclusions based on detailed comparisons between theoretical predictions and observations can not be taken seriously until it is ascertained that the theoretical predictions have used the correct boundary conditions. Further work, observational and theoretical, on the physical properties of coronal holes is clearly necessary for further progress in understanding the solar wind.

\section{Flow Geometry}

Almost all theoretical models of the solar wind expansion assume spherical symmetry, and radial expansion in flow tubes whose geometry increases as $r^{2}$. But even casual inspection of optical or X-ray photographs of the corona strongly suggests that the flow tubes expand considerably faster than $r^{2}$ for the first several solar radii above the solar surface. In addition, in situ observations, as well as coronal photographs, clearly indicate that the solar wind flow is not spherically symmetric. A problem similar to that described at the end of the preceding section thus presents itself, viz: conclusions based on comparisons between theoretical predictions and observations can not be taken seriously until realistic flow geometries and asymmetries are included in the theoretical calculations.

An important step in this direction has been made recently by Durney and Pneuman (1975), who attempted a calculation of the solar wind flow in a magnetic field geometry which was determined from the potential equations, using as boundary conditions actual magnetograph observations of the photospheric magnetic field. The calculated results did show structures at $1 \mathrm{AU}$ similar to those actually observed at the times corresponding to the magnetograph observations. But comparisons between the calculations and observations showed strong disagreement in detail. The disagreement was apparently caused by too rapid expansion of the magnetic field near the Sun, probably indicating that future work will require considerably more complex magnetic field calculations than the simple potential calculation employed by Durney and Pneuman. Refinements on their approach offer great promise, however. 
IV. Skylab

The principal contribution of Skylab to solar wind physics is the discovery of the so-called coronal transients, i.e. major large-scale rearrangements of the coronal plasma and magnetic field resulting from the sudden ejection of mass and magnetic flux into the solar wind (Gosling et al., 1974). The transients appear to be associated with eruptive prominences, and only rarely with flares. Their primary consequences for solar wind physics are: (i) the transients occur on the order of once every few hours, implying that the usual theoretical assumption of time-stationarity may frequently be inappropriate and that conclusions based on comparisons between observations and time-stationary theories may frequently be meaningless; (ii) the mass, energy, and momentum carried into the solar wind by a coronal transient may be locally quite significant, but relatively unimportant when averaged over a long time span and over the solar wind as a whole; (iii) the coronal transients may account for some, but not all, of the disturbed conditions which are frequently observed in the solar wind; in particular, they may account for the so-called 'magnetic bottles' in the solar wind, whose existence has been previously surmised by a variety of arguments.

\section{Physical Processes}

\section{MHD Waves}

One of the major advances of recent years has been the discovery that Alfven waves frequently dominate the fluctuations which are observed in the solar wind at 1 AU (Belcher et al., 1971; Belcher and Davis, 1971; Burlaga and Turner, 1975). The waves are most of ten observed to be propagating outward from the Sun, and a solar source, the supergranulation, has been suggested (Hollweg, 1972). The waves are observed to be preferentially associated with the high-speed solar wind streams at $1 \mathrm{AU}$, and they are frequently nearly circularly polarized. Their observed energy flux, when extrapolated back to the Sun, has been estimated to represent at least $15 \%$ of the total solar wind energy flux at the Sun.

These waves represent a natural laboratory for studying the propagation of large-amplitude MHD waves under collisionless conditions - a fact which may be hoped to pay dividends in other areas of astrophysics. Topics of recent interest in this regard are: (i) the nonlinear origin of the circular polarization at $1 \mathrm{AU}$; (ii) nonlinear collisionless damping of the waves; (iii) nonlinear evolution of the waves into shocks and discontinuities (e.g., Lee and Völk, 1973; Barnes and Hollweg, 1974; Cohen and Kulsrud, 1975). As yet no concensus has been reached as to which physical processes are the most important in determining the evolution of the waves as they propagate outward from the sun. But it can be hoped that this situation will be changed by continuing theoretical efforts coupled with the availability of new spacecraft data both closer to and farther from the sun than has heretofore been available. The question of the wave damping mechanism is particularly important, since wave damping may contribute in a major way to the physical development of the solar wind.

Alfvén waves may also contribute to the resolution of a number of theoretical difficulties facing solar wind theorists. Principal among these is the fact that the solar wind protons at $1 \mathrm{AU}$ are both hotter and faster than is predicted by reasonable theoretical models. The suggestion has accordingly been made that the difficulty can be resolved by introducing MHD waves of solar origin, which both heat and accelerate the solar wind protons. Theoretical models which include Alfvén waves (Hollweg, 1973) or fast mode MHD waves (Barnes et al., 1971) have yielded promising results which exhibit improved agreements with the observations.

Another aspect of the physical development of the solar wind which may be clarified with the aid of Alfvén waves concerns the flow of minor ions, particularly helium nuclei. The principal unexplained discrepancies between theory and observation are: (i) the helium ions almost always flow at least as fast as the protons at $1 \mathrm{AU}$, and sometimes faster, in contrast to most reasonable theories which give $V_{\mathrm{He}} \cong 0.75 V_{p}$ (ii) $T_{\mathrm{He}}>T_{p}$ (iii) $N_{\mathrm{He}} \mid N_{p} \cong 0.04$; in contrast to most reasonable theories which predict much smaller values for $N_{\mathrm{He}} \mid N_{p}$. Alfvén waves have recently been used to preferentially accelerate the helium nuclei in the solar wind, and some 
partial improvement in points (i) and (ii) has resulted (Hollweg, 1974; Turner and Hollweg, 1975). But the problems must be considered far from resolved. It is hoped that continued progress will be made in this area, especially since it seems likely that the behavior of helium nuclei in the solar wind can offer valuable clues to a variety of important physical processes occuring in the corona and solar wind.

It must be emphasized, however, that the necessity of introducing MHD waves into solar wind models can not be adequately assessed until such question as the boundary conditions, the flow geometry, time-stationarity and the correct electron energy equation (Section C.II) are adequately resolved. In addition, MHD waves can not positively be regarded as an additional energy source to the solar wind until they are proven to be of external (i.e. solar) origin, a question for which observational support is lacking.

\section{Electrons}

Until very recently, solar wind models have primarily used the classical Spitzer-Härm expression for the electron thermal conductivity. But it has also been known for a long time that the Spitzer-Härm conductivity can not be correct in the solar wind, because: (i) its use leads to results which disagree with observations at $1 \mathrm{AU}$; (ii) its use leads to results which are inconsistent with its derivation. Similar remarks apply also to the classical expressions for electron-ion thermal coupling.

Recent progress in describing the electron heat conduction has centered around two competing notions: (i) 'collisionless electron heat conduction' similar to what would pertain if the solar wind electrons were strictly collisionless (Hollweg, 1975); (ii) electron heat conduction controlled by plasma instabilities which are driven by the heat conduction itself (Forslund, 1970; Hollweg, 1974; Feldman et. al., 1974). There is probably some truth to both of these viewpoints, since observations of electron distribution functions at 1 AU (e.g. Feldman et al., 1974) indicate the presence of two electron populations, one of which is apparently controlled by instabilities while the other may be essentially collisionless. In either case, the heat conduction can not be written as a conductivity multiplied by a temperature gradient: more general forms are required.

The consequences of the collisionless electron heat conduction have already been explored in a simple solar wind model. Significant improvements in the agreement between theory and observation result, suggesting that it may be possible to construct realistic solar wind models without the aid of MHD waves of solar origin.

On the other hand, a proper understanding of the role of instabilities in controlling the heat conduction is at present not available. What will be required is, at very least, a quasi-linear theory for wave-particle interactions in a non-homogeneous flowing solar wind. Such a calculation lies in the future, but a step in that direction has been pursued by Gary et al. (1975), who have identified the instabilities which may be most effective in regulating the electron heat conduction.

Little work has been accomplished in describing the electron-ion thermal coupling, but ion damping or electron-driven instabilities must play a crucial role.

\section{Distribution Functions}

A major observational advance with significant theoretical consequences is the recent improvement in the quality of published distribution functions of electrons, protons, and helium nuclei in the solar wind (Feldman et al. 1973, 1974). Particle distribution functions represent a more advanced level of observational data than merely the moments of the distribution functions. Whereas most previous solar wind models have only concentrated on explaining the observed moments, it will now become necessary to explain the observed distribution functions as well. Since the observed distribution functions of all species show evidence of being influenced by waves and instabilities of various types, one will need at least a quasi-linear theory of wave-particle interactions in a non-homogeneous flowing solar wind, which is not yet available. The observed distribution functions often take peculiar forms (e.g. 
the electrons frequently consist of two anisotropic Maxwellians, one cool and dense, the other hot and rarefied, which drift relative to one another along the interplanetary magnetic field; the protons frequently show two peaks, with the anisotropy of the 'core' in a sense opposite to the anisotropy of the distribution as a whole), and their explanation represents a formidable problem. But at the same time they are providing valuable clues to a variety of plasma processes which, when identified and understood, will greatly enhance our understanding not only of the solar wind as a whole, but of plasma physics in general.

\section{REFERENCES}

Bame, S. J.: 1972, 'Spacecraft Observation of the Solar Wind Composition', NASA-SP-308, 535.

Barnes, A.: 1972, 'Microscale Fluctuations in the Solar Wind', NASA-SP-308, 333.

Barnes, A.: 1974, Adv. Electronics Electron. Phys. 36, 1.

Beckers, J. M.: 1972, Ann. Rev. Astron. Astrophys. 10, 73.

Burlaga, L. F.: 1971, Space Sci. Rev. 12, 600.

Burlaga, L. F.: 1972, 'Microstructure of the Interplanetary Medium', NASA-SP-308, 309.

Davis, L.: 1972, 'The Interplanetary Magnetic Field', NASA-SP-308, 93.

Hirshberg, J.: 1973, Rev. Geophys. Space Phys. 11, 115.

Hollweg, J. V.: 1974, Publ. Astron. Soc. Pacific 86, 561.

Hollweg, J. V.: 1975, Rev. Geophys. Space Phys. 13, 263.

Hundhausen, A. J.: 1972, Coronal Expansion and Solar Wind, Springer-Verlag, New York.

Lemaire, J. and Scherer, M.: 1973, Rev. Geophys. Space Phys. 11, 427.

Montgomery, M. D.: 1972, in Cosmic Plasma Phys. 61.

Newkirk, G.: 1972, 'Coronal Magnetic Fields and the Solar Wind', NASA-SP-308.

Parker, E. N.: 1971, Rev. Geophys. Space Phys. 9, 825.

Scarf, F. L.: 1970, Space Sci. Rev. 11, 234.

Schatten, K. H.: 1975, Rev. Geophys. Space Phys. 13, 589.

Stein, R. F. and Leibacher, J.: 1974, Ann. Rev. Astron. Astrophys. 12, 407.

Völk, H. J.: 1975, Space Sci. Rev. 17, 255.

\section{LARGE SCALE STRUCTURE OF THE SOLAR WIND}

\section{(S. T. Suess)}

\section{A. Introduction}

The interplanetary medium continues to serve as an important object of the experimental and theoretical interest with current studies of its own dynamics and structure, and with efforts to apply what is learned to understanding the sun and astrophysical plasma dynamics. The vast majority of our knowledge of the solar wind is limited to the ecliptic plane and $1 \mathrm{AU}$ from the Sun. However, the last few years have produced a great expansion in our knowledge of properties out to $5 \mathrm{AU}$ and beyond (by Pioneers 10 and 11 ), inwards to $0.31 \mathrm{AU}$ (by Mariner 10 and Helios), and out of the ecliptic through interplanetary scintillation (IPS) and comet tail observations. This has been naturally accompanied by increased efforts in general global modelling of the solar wind, extending earlier work and concepts to include the new areas of knowledge. Because of intrinsic limitations, little of this work deals with kinetic models (Lemaire and Scherer, 1973), but rather fluid models - even though little progress has been made in the fundamental understanding of why fluid models work so well.

\section{B. Steady - State, Axisymmetric Flow}

Considerable effort has been expended in attempting to describe radial and meridional gradients in the solar wind, exclusive of any temporal or azimuthal variations. Simultaneous measurements of two spacecraft (Lazarus et al., 1970; Intriligator and Neugebauer, 1974), time series measurements by spacecraft moving inward or outward in the solar system (Wolfe, 1974; Neugebauer, 1975), IPS studies (Coles et al., 1974), and comet observations (Brandt et al., 
1975) all provide useful information. Results are consistent with no radial gradient in the radial velocity from 0.3 to more than $5.0 \mathrm{AU}$, with an uncertainty of less than $10 \%$. At the present, there is little new information on the angular momentum flux, although Helios data could soon change this. The only available analysis (Lazarus and Goldstein, 1971) shows approximately constant angular momentum flux between 0.7 and 1.0 AU. The mass flux in the ecliptic also seems to agree with an inverse square dependence of flux on radius, although the uncertainty at large distances is large. The temperature is the most poorly measured parameter in the outer solar system, and the Helios results are not yet in. However, Pioneer 10 results (Neugebauer, 1975) suggest the proton temperature falls off at a rate less than $r^{-4 / 3}$, perhaps about $r^{-1}$. Gringauz (1975) has reported that the electron temperature varies as $r^{-1 / 2}$ between Mars and $1 \mathrm{AU}$.

The situation is not clear with regard to latitudinal variations. IPS data show consistent increases in radial velocity with latitude, between $60^{\circ} \mathrm{S}$ and $80^{\circ} \mathrm{N}$, of about $2 \mathrm{~km} \mathrm{~s}^{-1} \mathrm{deg}^{-1}$ of latitude with some possibility of long term or solar cycle variations. In situ measurements in the ecliptic show radial velocity gradients of up to $15 \mathrm{~km} \mathrm{~s}^{-1} \mathrm{deg}^{-1}$ (Rhodes and Smith, 1974) - again with some possibility of a solar cycle variation. Furthermore, the inverse density-velocity correlation seems to be preserved in these observations. Comet observations throw some uncertainty into the interpretation of latitudinal velocity gradients. Brandt et al. (1975) report no latitudinal or radial variation in the radial velocity from observations extending over several decades but, necessarily, averaged over that period. They also report an azimuthal velocity varying as $(\cos b)^{\mathrm{n}} / r(b=$ latitude, $n=2.315)$, which is consistent with in situ results.

Essentially all models of the solar wind predict a small or zero gradient of the radial velocity with radius beyond a few tens of solar radii, but are conflicting on the questions of temperature variation and magnitude. Durney and Hundhausen (1974) arrive at an adiatatic gradient beyond a few $\mathrm{AU}$, but have rather large electron temperature at $1 \mathrm{AU}$. Their model could also be interpreted as suggesting a temperature decrease with latitude, Leer and Axford (1972), on the other hand, predict increasing temperature with latitude. The results depend largely on initial assumptions about gradients near the Sun. Hydromagnetic effects (Winge and Coleman, 1974) can contribute slightly to the radial velocity, to latitudinal gradients in the radial velocity, and to the temperature variation; but are insufficient to account for the observations. A similar quasi-radial flow model (Suess and Nerney, 1975) permits large latitude variations in radial velocity, but has not been carried so far as the above.

Solar cycle effects have long been inferred from cosmic ray and geomagnetic variations. However, Diodata et al. (1974) now find direct variation in the density. Hirshberg (1973) has also suggested a solar cycle dependence of the velocity distribution and modal value. However, the proton bulk velocity is essentially constant and the proton temperature shows no clear trend.

\section{Interplanetary Magnetic Field}

The radial magnetic field component has been found to fall off very nearly as $r^{-2}$, whereas the azimuthal component seems to fall off more rapidly than $r^{-1}$. This is a contradictory result as the former agrees with classical theory and the latter does not. Proposals to explain this are stream interaction (Hundhausen, 1972) and the MHD effects mentioned above (Nerney and Suess, 1975). However, the first is only a hypothesis and the second effect tends to modify both components of the field similarly. The topology of the interplanetary magnetic field (IMF) has been further defined (Wilcox and Colburn, 1972; Rosenberg and Coleman, 1969). The width of sectors depends on latitude, suggesting a field represented by the superposition of an axial and an equatorial dipole (Svalgaard et al., 1975). Furthermore, the sector structure shows distinct solar cycle effects in number and width, with one component rotating with a fixed period over five solar cycles. These results have shown the IMF to be a rich source of information on the large scale structure of the solar magnetic field. 


\section{Nonradial Flow}

Nonradial flow imposed near the sun, by the coronal structure, falls off so rapidly with distance as to be insignificant at the 1 AU (Sroczynska, 1974), although it can lead to large errors in tracing a structure observed at 1 AU back to the Sun. The earlier mentioned MHD effects drive a meridional flow, redistributing mass and magnetic flux in latitude. For simple boundary conditions, the flow is away from the equator and produces approximately a $10 \%$ equatorial flux deficit between 1 and $5 \mathrm{AU}$. The meridional flow varies as cos $(2 b)$, with an amplitude of a few $\mathrm{km} \mathrm{s}^{-1}$. Brandt et al. (1973) find evidence for such a flow in comet data. A meridional component of the IMF away from the equator is reported by Coleman and Rosenberg (1971). Lazarus and Goldstein (1971) report a flow direction towards the equator in 1967. Heating of the plasma by interstellar neutral gas can also produce a nonradial flow away from the inflow direction and of several $\mathrm{km} \mathrm{s}^{-1}$ (Grzedzielski and Ratkiewicz, 1975), but this would be difficult to observe in the inner solar system.

\section{E. Streams and Shocks}

The Pioneer-10 and -11 results, along with several other space probes, indicate corotating streams steepen with distance into shocks with these shocks continuing essentially undiminished into the outer solar system. The tendency is for the longer streams to envelope all smaller ones. The velocity jumps decrease with distance (Collard and Wolfe, 1974), but the mach number seems to increase. This is in agreement with numerical models (Hundhausen, 1973), which predict over a 90\% momentum flux density enhancement in the compression region of the streams beyond $5 \mathrm{AU}$. Stream topology out of the ecliptic has been observed with the IPS technique (Houminer, 1973) and modelled by Suess et al. (1975). Nonradial flow and MHD effects on large amplitude corotating streams have not been satisfactorily investigated, so that large changes in flow direction (Burlaga, 1975) and plasma structure (Feldman, 1974) are not understood. The passage of streams at $1 \mathrm{AU}$ has been found to be correlated with Forbush decreases (Barouch and Burlaga, 1975) and it has been proposed that the compression of the IMF by streams could produce the desired effects through $\mathbf{E} \times \mathbf{B}$ drifts of the cosmic ray particles.

Numerical models of hydrodynamic propagation have also succesfully represented observed shock dynamics (Dryer, 1975). Recently, magnetic effects in large amplitude shocks have also been modelled by Nakagawa and Welck (1973) and Steinolfson (1975). It is an advantage that the equations representing shock propagation are much the same as those for stream propagation. It has been found that shocks propagating in interacting streams are strongly distorted (Hirshberg et al., 1974; Krimsky and Transky, 1974), explaining much of the earlier nonradial shock normal observations. Many flare produced shocks are also accompanied by non-corotating streams (Burlaga, 1975), but the source of these streams is poorly understood. The similarity of stream interactions and shock formation and propagation to time dependent astrophysical flows is striking.

\section{F. Other Topics}

Alpha particles and heavier ions are still a mystery. The alpha to proton number density ratio has been shown to vary with solar velocity (Ogilvie and Hirshberg, 1974) and, consequently, with latitude and position in streams. However, this correlation is not understood any more than the $\alpha$-particle velocity and temperature structure. Better models of the transition from the corona will also allow a better evaluation of momentum loss from the Sun when coupled with already available models for the interplanetary flow.

\section{REVIEW ARTICLES}

Barnes, A.: 1974, in L. Marton (ed.) Electronics Electron Phys. 36, 1. Dobrowolny, M. and Moreno, G.: 1975, Space Sci. Rev. (in press). 
Dryer, M.: 1975, Space Sci. Rev. 17, 277.

leeldman, W. C., Asbridge, J. R., Barnes, S. J. and Montgomery, M. D.: 1974, Rev. Geophys. Space Phys. 12, 715.

Hollweg, J. V.: 1975, Rev. Geophys. Space Sci. 13, 213.

Hundhausen, A. J.: 1972, Coronal Expansion and the Solar Wind, Springer-Verlag, New York.

Montgomery, M. D.: 1973, Space Sci. Rev. 14, 559.

Lemaire, J. and Scherer, M.: 1973, Rev. Geophys. Space Sci. 11, 427.

Neugebauer, M.: 1975, Space Sci. Rev. 17, 221.

Russell, C. T. (ed.):1974, Solar Wind Three (Proc. of the Third Solar Wind Conf., Asilomar, Calif., Mar. 1974), Inst. of Geophys. and Planet Phys., Univ. Calif. Los Angeles.

Sonett, C. P., Coleman, P. J. and Wilcox, J. M. (eds.): 1972, Solar Wind (Proc. of the Second Solar Wind Conf., Asilomar, Calif., Mar. 1971), NASA-SP-308. U.S. Nat. Rept. to the IUGG: 1975, Rev. Geophys. and Space Sci. 13, No. 3.

\section{THE DISTANT SOLAR WIND AND NEARBY INTERSTELLAR MATTER}

(P. W. Blum and H. J. Fahr)

\section{A. The Interstellar Wind}

The neutral component of the interstellar medium is able to penetrate almost unimpeded into the heliosphere since it is not affected by the interplanetary magnetic field nor does the neutral interstellar gas suffer appreciable deflections of its flow due to collisions with interplanetary particles. The direction relative to the sun from which the interstellar wind enters the solar system can be deduced by an analysis of the distribution of the scattered interplanetary solar resonance radiations. Based on the distribution of scattered solar Lyman- $\alpha$ radiation this direction has been found to be $\alpha=265^{\circ}, \delta=-22^{\circ}$ (1). Weller and Meier $(2,3)$ have show $n$ that based on the interplanetary He-584 $\AA$ resonance radiation this direction is $\alpha=252^{\circ}, \delta=-15^{\circ}$. No physical explanation that accounts for this difference of $13^{\circ}$ has been given until now. Both these results show, however, that the direction of the interstellar wind differs appreciably from the solar apex direction. This proves that the interstellar medium in the vicinity of the solar system has a peculiar motion relative to the local standard of rest of nearby stars. Furthermore, the velocity vector of the interstellar wind is nearly in the ecliptic plane, but this seems to be purely coincidental.

Recently it has been shown (4) that if a latitudinal variation of the solar wind is assumed to exist, then the derivation of the direction of the interstellar wind velocity becomes uncertain. Part of the neutral interstellar atoms are ionized inside the heliosphere by solar wind particles and the solar EUV radiation. The ionization rates for $\mathrm{H}, \mathrm{He}, \mathrm{C}, \mathrm{O}, \mathrm{Ne}, \mathrm{Si}, \mathrm{Ar}$, and $\mathrm{Fe}$ have been deduced by Johnson (5) from the relevant cross sections of the various processes and the intensities of the solar EUV- and corpuscular fluxes. Interplanetary neutral hydrogen is mainly ionized by charge-exchange reactions with solar wind protons. This interaction amounts to about $70 \%$ of all ionization processes for interplanetary hydrogen. EUV ionization accounts for only about $20 \%$. For helium the importance of charge-exchange and EUV ionization is more than reversed; namely the EUV ionization rate is almost $95 \%$ of the total ionization rate. The contribution of impact ionization by solar wind electrons is generally less than $10 \%(6)$. The total ionization rate at $1 \mathrm{AU}$ from the sun is of the order of $6.10^{-7} \mathrm{~s}^{-1}$ for hydrogen and one order of magnitude less for helium (5).

\section{B. Distribution of the Neutral Atoms Inside the Heliosphere}

The distribution of the interstellar neutrals inside the heliosphere is determined by the ionization rate of the particular atomic species and the focusing effect of the heliocentric force acting on the atoms. For all species except for hydrogen the only heliocentric force is the solar gravitation. For hydrogen the solar $L y-\alpha$ radiation pressure cannot be neglected since it is of the same magnitude as the solar gravitation. A solar Ly- $\alpha$ flux of approximately $6 \mathrm{erg} \mathrm{cm}^{-2} \mathrm{~s}^{-1}$ at $1 \mathrm{AU}$ suffices to balance completely the solar gravitation. According to observations (7) of the 
solar Ly- $\alpha$ emission one would expect either a balancing of the attractive and the repulsive forces or even a considerable repulsion (1) due to an excess of the radiation pressure over the gravitational force. Thus neutral hydrogen is not able to penetrate into the downwind region at all. Interplanetary helium is not subjected to any radiation pressure comparable to the gravitational force. Therefore the helium density is considerably enhanced in the downwind direction due to gravitational focusing.

Analytic and numeric models of the distribution of the neutral interplanetary gases have been calculated by several authors $(1,5,6,8,9)$. A good analytic approximation for the neutral density on the downwind axis has also been deduced (10) for helium and heavier atoms. The distribution of the neutrals inside the heliosphere is determined to a considerable extent by the state of the interstellar medium just outside the heliosphere. Its absolute density, its velocity relative to the Sun and its temperature are the parameters that enter the interplanetary density distribution function. The interplanetary distribution of the heavier elements like $\mathrm{C}, \mathrm{O}, \mathrm{N}$ and $\mathrm{Ne}$ can in principle be deduced from the distribution of hydrogen and helium, but it must be taken into account that the interstellar density ratio of the various neutral elements may not be equal to the cosmic abundance ratio because the various neutral species have different ionization ratios in the interstellar space $(11,12)$.

The density of neutral hydrogen outside the solar system is deduced from the scattered interplanetary Ly- $\alpha$ radiation. The densities that are obtained by the various authors scatter between 0.03 and $0.12 \mathrm{~cm}^{-3}$, depending on the basic assumptions that enter into the models. It has been suggested (12) that multiple scattering of Ly- $\alpha$ photons cannot be neglected. Based on a model which incorporates multiple scattering, Wallis has obtained a neutral hydrogen density of $0.3 \mathrm{~cm}^{-3}$. Recently it has become possible to observe the Ly- $\alpha$ emission line from a star only $3.5 \mathrm{pc}$ from the Sun. A neutral hydrogen density of only $0.015-0.03 \mathrm{~cm}^{-3}$ (14) was derived from the analysis of this line.

The velocity of the interstellar wind relative to the sun is difficult to ascertain as no direct observations have been possible, and therefore the only way is to compare the observed interplanetary resonance radiation with a model of the neutral density distribution. Velocities between $5-30 \mathrm{~km} \mathrm{~s}^{-1}$ fit the observations $(2,15,16)$. A similar, if not even greater uncertainty applies to the determination of the temperature of the interstellar medium in the vicinity of the solar system. Based on the idea that the solar system is embedded in the tenuous hot intercloud medium, one would expect an interstellar temperature between 1000-10000 K. Bertaux et al. (17) have found temperatures between $5800 \mathrm{~K}$ and $13000 \mathrm{~K}$.

The charge-exchange reactions between interplanetary neutral hydrogen and solar wind protons produce protons which have the slow interstellar wind velocity and neutral hydrogen atoms that have the fast solar wind velocity. The latter move approximately radially outwards from the heliosphere. They are not subjected to solar radiation pressure effects because their radial velocity is too high. These fast neutrals have a density maximum between 1 and $10 \mathrm{AU}$, where their density may reach $10^{-3} \mathrm{~cm}^{-3}$. Furthermore, their distribution in interplanetary space shows a broad maximum in the upwind direction. A possible contribution of these fast neutrals to the Lyman- $\alpha$ background radiation is under investigation (18).

\section{The Distant Solar Wind}

Advancing from smaller to larger solar distances, the characteristic expansion time of the solar wind decreases monotonically. Compared to this increase the collision times of solar wind electrons and protons show steeper increases inside of about $10^{2}$ solar radii, but flatter increases beyond. This is due to the fact that the collision frequencies drop with decreasing solar wind densities, but at the same time increase with falling temperatures, since the Debye shielding of charged species within the solar wind plasma determining the Coulomb collision cross sections is less effective at lower temperatures. This results in a crossover of the characteristic expansion times and the collision times at about $10^{3}$ solar radii. Between $10^{2}$ and $10^{3}$ solar radii we have characteristic expansion times and electron collision times of comparable orders of magnitude. Hence we do not expect to find anisotropic electron temperatures there. In the region inside of 500 solar radii the collision times of protons are 
larger than the characteristic expansion times by about one order of magnitude. This yields approximately the situation of a collisionless solar wind proton plasma. Since also the thermal conduction of protons and electrons becomes negligible beyond $1 \mathrm{AU}$, this results more or less in an adiabatic expansion of the solar wind plasma with sharply decreasing temperatures $T_{p}$ of protons and $T_{e}$ of electrons.

In the collision-free proton plasma anisotropic temperatures will develop with higher thermal spreads $\left(T_{\|}\right)$parallel to the magnetic field of the solar wind rather than $\left(T_{\perp}\right)$ perpendicular to it. Outside the orbit of the Earth where the frozen-in magnetic field of the solar wind plasma tends to become a purely azimuthal field, the thermal spread in radial directions decreases nearly adiabatically due to the bad thermal conduction perpendicular to the field. Contrary to this, the thermal spread in azimuthal directions behaves much more like in the isothermal case due to the relatively high parallel conductivities. (9)

Beyond the crossover point at about $10^{3}$ solar radii again the characteristic expansion times become larger than the collision times of solar wind electrons and protons. Therefore the temperatures of both the electrons and the protons tend to become again isotropic, and the solar wind expansion in this distant solar region is again collision-dominated.

Generally only steady state solar wind expansion models have been developed. Hundhausen (19) has studied numerically the passage of a solar wind perturbation due to a step-like variation of the coronal boundary conditions. This investigation shows how a pressure pulse at the coronal base of a few hundred hours duration propagates through the steady solar wind. The propagation of such a pressure pulse is used as a simplified model of the interaction of often-observed high speed solar wind streams with the steadily flowing quiet solar wind. Assuming this model represents the main features of a high speed solar wind interaction, it was shown that only a very minor fraction of the kinetic energy of the pressure pulse is irreversibly dissipated into the steady solar wind environment. The overwhelming majority of energy expenditure is a reversible adiabatic heating effect during the compression time of the pulse passage. This has also been confirmed by the solar wind data analysis of Burlaga and Ogilvie (19), who investigated the relation between proton temperature $\left(T_{p}\right)$ and the solar wind velocity $v_{s}$ for phases of negative and positive velocity derivatives $\mathrm{d} v_{s} / \mathrm{d} t$, i.e. immediately before and after the passage of a high velocity flow. Therefore it may not be expected that the interaction of high speed flows with the steady solar wind will result in higher temperatures of the distant solar wind than given by the theoretical steady solar wind models.

As long as the solar wind plasma can be considered to be a perfect electrical conductor, the concept of frozen-in magnetic fields moving with the solar wind plasma is valid, and magnetic field lines cannot reconnect. Therefore adjacent regions of oppositely directed magnetic fields remain separated by neutral sheets (20). The parameter $\beta$ which gives the ratio of thermal over magnetic field energy densities is a decreasing function of the solar distances in regions of near adiabatic plasma cooling and azimuthal magnetic fields. This means that in the distant solar wind we may well find regions of very low values of $\beta$. This results in a tendency to a breakup of magnetic sector boundaries and field line reconnections, meaning that in the distant solar wind we may find isolated magnetic islands of self-consistently expanding plasma enclosures.

\section{Change of the Distant Solar Wind Parameters due to Interactions with the Neutral Interplanetary Gas}

Most of the solar wind expansion models treat the solar wind plasma as if it would be expanding into a circumsolar vacuum. This is of course not true, since the solar system is surrounded by the interstellar gas, whose neutral component can penetrate deeply into the solar system. Comparing the energy densities of the neutral interstellar gas and the solar wind plasma, it is evident that the interaction of these media cannot lead to a change of the solar wind dynamics within solar distances less than $3 \mathrm{AU}$. Outside of this region the energy density of the neutral interstellar medium is, however, comparable or even larger than that of the solar wind plasma. Therefore beyond $3 \mathrm{AU}$ it is unavoidable to consider the effect of an interaction of these two media on the density, velocity and temperature of the solar wind. The interaction takes place via charge-exchange collisions and EUV ionizations of neutral interstellar 
constituents leading to the production of secondary ions that are integrated into the original solar wind plasma regime. This leads to additional terms in the differential equations of mass, momentum and energy continuity. The effects of these terms have been studied by Holzer (21), Wallis $(22)$ and Fahr $(23,24)$. In short these papers show that the distant solar wind instead of keeping a constant asymptotic expansion velocity is steadily decelerating due to an exhaustion of linear momentum by neutralized solar wind ions.

Furthermore, there is a decrease of the solar wind densities flatter than $1 / r^{2}$ due to the integration of additional secondary ions into the solar wind plasma. Finally, we expect a strong enhancement of the temperatures from their vacuum solution values due to suprathermal ions that heat up the solar wind plasma. Depending on the mechanism of thermal coupling between thermal and suprathermal solar wind ions, an increase of solar wind temperatures with increasing distances outside of about $3 \mathrm{AU}$ may even result.

\section{E. The Termination of the Heliosphere}

Since the solar wind is expanding into a surrounding interstellar medium characterized by neutral and ionized constituents as well as interstellar magnetic fields of a probable order of $3 \times 10^{-6} \mathrm{G}$, there must be a region somewhere outwards from the Sun where the energy densities of the solar wind plasma and the in terstellar medium balance. At these solar distances the undisturbed, self-consistent expansion of the solar wind terminates, defining the so-called heliopause. Inside of the heliopause is the region of the solar wind domain, i.e. the heliosphere.

The location of the point of balancing is strongly affected by the heliospheric interaction of the neutral interstellar gas component with the solar wind. This interaction leads to an energy exhaustion of the solar wind plasma, as previously mentioned. This influence of the neutral interstellar gas component on the location of the point of energy balancing has been discussed by Fahr (25), Semar (26) and Axford (9). It was found that the point of balancing where a shock transition of supersonic to subsonic solar wind expansion is supposed to occur $(21,22)$ is shifted from about a solar distance of $180 \mathrm{AU}$ to about $50 \mathrm{AU}$ for a variation of the interstellar neutral gas density from 0 to 1 hydrogen atom $\mathrm{cm}^{-3}$. Beyond this point the expanding solar wind is assumed to be subsonic. Its further expansion has to become adapted to the surrounding interstellar field, which is most likely to be homogeneous in the solar vicinity. The main features of the possible forms of this adaption have been discussed by Parker (27). In order to describe the adaptation to the external interstellar field he assumes the undisturbed heliosphere to be a purely diamagnetic sphere which perfectly withstands penetration of interstellar field lines. This requires the boundary condition of a purely tangential field at the surface of the diamagnetic sphere. The solution of a Poisson equation for the magnetic field potential yields a description of the magnetic surroundings of the heliosphere. The assumption, however, is that there are no electrical currents outside of the heliosphere. Bird and Fahr (28) proposed an addition to this description by taking into account currents along an elliptically shaped diamagnetic plasmoid. These currents lead to the outer magnetic field surroundings of the plasmoid. The elliptical shape of the plasmoid is also self-consistently determined by considering the fact that the solar wind plasma expansion perpendicular to the ambient interstellar field is impeded, whereas the expansion parallel to the ambient field is not. As has been shown in a review article by Axford (9), the shape of the solar wind plasmoid is not exclusively determined by a plasma-field interaction, but in addition by a 'plasma-plasma' and 'plasma-neutral gas' interaction. The latter case of an interaction between the expanding distant solar wind and the homogeneous flow of the interstellar gas leads to a solar wind plasmoid similar to the tail of a comet. The real shape of the solar wind plasmoid will therefore be a complicated mixture of all these interaction types. The combined interaction of interstellar fields and gases with the solar wind plasma has up to now not been theoretically described.

\section{REFERENCES}

(1) Thomas, G. E.: 1973, 'The Neutral Interplanetary Medium', Paper 73-547 at the AIAA/AGU Space Science Conference on the Exploration of the Outer Solar System, Denver, Colorado, July 10-12, 1973. 
(2) Weller, C. S. and Meier, R. R.: 1974, Astrophys. J. 193, 471.

(3) Weller, C. S. and Meier, R. R.: 1975, submitted to Astrophys. J.

(4) Joselyn, J. A. and Holzer, T. E.: 1975, J. Geophys. Res. 80, 903.

(5) Johnson, H. E.: 1972, Planetary Space Sci. 20, 829.

(6) Blum, P. W., Pfleiderer, J., and Wulf-Mathies, C: 1975, Planetary Space Sci. 23, 93.

(7) Vidal-Madjar, A., Blamont, J. E., and Phissamay, B.: 1973, J. Geophys. Res. 78, 1115.

(8) Blum, P. W. and Fahr, H. J.: 1970, Astron. Astrophys. 4, 280.

(9) Axford, W. I.: 1972, in C. P. Sonnett, P. J. Coleman, Jr, and J. M. Wilcox (eds.) The Solar Wind, NASA SP-308, p. 609.

(10) Feldman, W. C., Lange, J. J., and Scherb, F.: 1972, in C. P. Sonnett, P. J. Coleman, Jr., and J. M. Wilcox (eds.) The Solar Wind NASA SP-308, pp. 684.

(11) Blum, P. W. and Fahr, H. J.: 1975, Astrophys. Space Sci. (in press).

(12) Wulf-Mathies, C. and Blum, P. W.: 1975, Space Res. XIV (in press).

(13) Wallis, M.: 1974, Monthly'Notices Roy. Astron. Soc. 167, 103.

(14) Evans, R. G., Jordan, C. and Wilson, R.: 1975, Nature 253, 5493.

(15) Thomas, G. E. and Krassa, R. F.: 1971, Astron. Astrophys. 11, 218.

(16) Bertaux, J. L. and Blamont, J. E.: 1971, Astron. Astrophys. 11, 200.

(17) Lang, R.: 1975, Private communication.

(18) Hundhausen, A. J.: 1973, J. Geophys. Res. 78, 1528.

(19) Burlaga, L. F. and Ogilvie, K. W.: 1973, J. Geophys. Res. 78, 2028.

(20) Axford, W. I.: 1967, In B. M. McCormac (ed.) Aurora and Airglow, Reinhold Publ. Co., New York.

(21) Holzer, T. E.: 1972,J. Geophys. Res. 77, 5407.

(22) Wallis, M.: 1973, Astrophys. Space Sci. 20, 3.

(23) Fahr, H. J.: 1973, Solar Phys. 30, 193.

(24) Fahr, H. J.: 1974, in Formisano (ed.) The Magnetospheres of Earth and Jupiter, Reidel, Dordrecht, p. 269.

(25) Fahr, H. J.: 1971, Planetary Space Sci. 19, 1121.

(26) Semar, C. L.: 1970, J. Geophys. Res. 75, 6892,

(27) Parker, E. N.: 1963, in Interplanetary Dynamical Processes, Interscience Publishers, New York.

(28) Bird, M. and Fahr, H. J.: 1973, "The Effect of the Interstellar Field on Size and Shape of the Heliosphere', Preprint, Univ. of Bonn.

I wish to thank Drs Blum, Fahr, Hollweg and Suess for their contributions to this report.

W. I. AXFORD

Acting President of the Commission 